\title{
Serial stereotactic biopsy of brainstem lesions in adults improves diagnostic accuracy compared with MRI only
}

\author{
W Rachinger, ${ }^{1}$ S Grau, ${ }^{1}$ M Holtmannspötter, ${ }^{2}$ J Herms, ${ }^{3}$ J-C Tonn, ${ }^{1}$ F W Kreth ${ }^{1}$
}

${ }^{1}$ Department of Neurosurgery, University of Munich, Munich, Germany; ${ }^{2}$ Department of Neuroradiology, University of Munich, Munich, Germany: ${ }^{3}$ Department of Neuropathology, University of Munich, Munich, Germany

Correspondence to: Professor Dr F W Kreth, Department of Neurosurgery, Klinikum Großhadern, Marchioninistr 15, 81377 Munich, Germany; friedrich.wilheln.kreth@med. uni-muenchen.de

W Rachinger and S Grau contributed equally to this paper.

Received 1 February 2009

Revised 12 May 2009

Accepted 18 May 2009

Published Online First

10 June 2009

\begin{abstract}
Objective: The aim of the current prospective study was to analyse the validity of MRI based diagnosis of brainstem gliomas which was verified by stereotactic biopsy and follow-up evaluation as well as to assess prognostic factors and risk profile.
\end{abstract}

Methods: Between 1998 and 2007, all consecutive adult patients with radiologically suspected brainstem glioma were included. The MRI based diagnosis of the lesions was made independently by an experienced neuroradiologist. Histopathological evaluation was performed in all patients from paraffin embedded specimens obtained by multimodal image guided stereotactic serial biopsy technique. Histopathological results were compared with prior radiological assessment. Length of survival was estimated with the Kaplan-Meier method and prognostic factors were calculated using the Cox model.

Results: 46 adult patients were included. Histological evaluation revealed pilocytic astrocytoma $(n=2)$, WHO grade II glioma $(n=14)$, malignant glioma $(n=12)$, metastasis $(n=7)$, lymphoma $(n=5)$, cavernoma $(n=1)$, inflammatory disease $(n=2)$ or no tumour/ gliosis $(n=3)$. Perioperative morbidity was $2.5 \%(n=1)$. There was no permanent morbidity and no mortality. All patients with "no tumour" or "inflammatory disease" survived. Patients with low grade glioma and malignant glioma showed a 1 year survival rate of $75 \%$ and $25 \%$, respectively; the 1 year survival rate for patients with lymphoma or metastasis was 30\%. In the subgroup with a verified brainstem glioma, negative predictors for length of survival were higher tumour grade $(p=0.002)$ and Karnofsky performance score $\leqslant 70(p=0.004)$.

Conclusion: Intra-axial brainstem lesions with a radiological pattern of glioma represent a very heterogeneous tumour group with completely different outcomes.

Radiological features alone are not reliable for diagnostic classification. Stereotactic biopsy is a safe method to obtain a valid tissue diagnosis, which is indispensible for treatment decision.

Intra-axial brainstem gliomas in adults represent approximately $2 \%$ of all brain tumours. ${ }^{1}$ In children, the proportion of these tumour is as high as $20-25 \% .{ }^{12}$ Several reports have dealt with brainstem gliomas in children in terms of their biological behaviour, MRI findings, prognostic factors and management..$^{3-9}$

In contrast, features and biology of brainstem gliomas in adults are less well known. Only a few reports have focused on this tumour entity with only one recent publication. ${ }^{10}$ In most of these studies, treatment decisions were based on MRI features alone and did not include a histopathological diagnosis. Most authors regard a biopsy of intrinsic brainstem tumours as too dangerous ${ }^{11-13}$ and consider imaging methods as sufficiently reliable. ${ }^{14}$ Thus the impact of MRI findings on treatment decisions for brainstem tumours is very high but the accuracy of MRI based diagnosis of brainstem gliomas has not to date been verified sufficiently by histopathological findings.

In the current literature, brainstem gliomas are differentiated by MRI characteristics into two subgroups: (a) focal tumours, which are typically well marginated on MRI, occupy less than $50 \%$ of the axial diameter of the brainstem and frequently show contrast enhancement. These tumours are usually regarded as low grade tumours and have a good prognosis; and (b) diffuse brainstem tumours, which are poorly marginated, rarely enhance with contrast and occupy more than $50 \%$ of the axial diameter of the brainstem. While the first group is normally interpreted as pilocytic glioma with a rather benign biology, the latter are mostly considered malignant gliomas. ${ }^{14}$

The aim of this prospective study was to analyse and validate the accuracy of conventional MRI based classifications of brainstem gliomas. This was achieved by comparing radiological diagnosis with histopathological findings. In this context, we also assessed risk profile as well as diagnostic and prognostic relevance of a tissue diagnosis obtained by stereotactic biopsy in patients with brainstem lesions. For evaluation of prognostic factors, length of survival was correlated with the obtained tissue diagnosis and all relevant pretreatment clinical and neuroradiological parameters.

\section{CLINICAL MATERIAL AND METHODS}

\section{Patients}

Between April 1998 and September 2007, all consecutive adult patients were included in this study if the epicentre of a suspected glioma was located in the brainstem. Patients with radiological and clinical evidence of disease other than glioma were excluded. MRI was evaluated with a lesion being suspicious of brainstem glioma (high or low grade) in all patients enrolled.

MRI based assessment and diagnosis was performed independently by an experienced neuroradiologist $(\mathrm{MH})$ twice, with and without knowledge of the clinical data (except for histology). According to current radiological guidelines, a brainstem glioma was defined by location of the lesion (ie, mesencephalon, pons, medulla oblongata) on imaging data (axial and sagittal T2 and T1 weighted images (with and without gadolinium)), 
performed with a $1.5 \mathrm{~T}$ scanner (Magnetom Vision; Siemens, Erlangen, Germany). Tumours were assessed according to current guidelines ${ }^{15}$ and distinguished as being focal (suspected low grade) or diffuse (suspected high grade). They were judged focal when they were well marginated, showing contrast enhancement and occupying less than $50 \%$ of the axial brainstem diameter. Poorly marginated tumours, with an extension of more than $50 \%$ of brainstem diameter, were classified as diffuse. The lesion was considered extended if it involved more than one segment of the brainstem (eg, medulla oblongata, pons).

For histological proof of diagnosis of brainstem glioma, all patients underwent stereotactic biopsy. MRI/CT guided stereotactic biopsy was performed as described previously. ${ }^{16} \mathrm{~A}$ modified Riechert Stereotaxy System (MHT, Freiburg, Germany) was used. Target definition for the biopsy procedure was based on MRI. CT and MRI images were fused on a Brain Lab work station. (Brain Lab image fusion software; Brain Lab, Heimstetten, Germany) A $6 \mathrm{~mm}$ Burr whole was made and serial biopsies along an exactly defined trajectory representative of the area of interest were taken using micro forceps. The average amount of tissue per biopsy specimen was $1 \mathrm{~mm}^{3}$ and the number of specimens taken in $1 \mathrm{~mm}$ steps ranged from 5 to 15 samples per patient. Smear preparations of the biopsy specimens were examined intraoperatively by an attending neuropathologist in all patients. After the neuropathologist's statement that the amount of specimen was sufficient for assessment, the biopsy procedure was stopped. The final diagnosis was made from paraffin embedded tissue specimens and the smear preparations $(\mathrm{JH})$. Additional immunohistochemical examination and an assessment of the proliferation index were used.

For immediate follow-up, each patient (a) received a CT scan postoperatively, which was assessed by a neuroradiologist to confirm stereotaxy marker position and exclude haemorrhage and (b) was examined clinically by a neurosurgeon for detection of procedure related symptoms. Clinical follow-up was in 3 month intervals with a minimum follow-up of 1 year for all patients. If patients did not attend for follow-up, the necessary information was obtained by phone interview. Median followup time for survivors was 33 months.

Treatment was initiated according to current recommendations and standards: radiotherapy for low grade gliomas and lymphomas, radiochemotherapy for high grade gliomas, radiosurgery for singular metastasis, antibiotic treatment for abscess, immunosuppression for autoimmune inflammatory disease and no treatment (observation) for no tumour/gliosis.

Sensitivity and specificity of conventional MRI imaging for diagnosis of brainstem gliomas was estimated by comparison with histological results.

\section{Statistical analysis}

Length of survival was estimated using the Kaplan-Meier method. The impact of pretreatment factors on survival was analysed using the Cox model.

\section{RESULTS}

\section{Patient population}

Between April 1998 and September 2007, 46 patients were included (25 men, 21 women). All patients were adults (>18 years). Age ranged from 18 to 78 years (median 43 years). Median follow-up was 17 months (range 1 month to
91 months) (table 1). Clinical signs included ataxia $(n=30)$, cranial nerve deficit $(n=27)$ and hemiparesis $(n=19)$.

\section{Surgical procedure and complications}

Three patients received a second biopsy (because of nonpathological findings after the first procedure) and thus 49 procedures were performed. The surgical approach was frontal extraventricular in 41 cases and suboccipital transpeduncular in eight cases. There were two procedure related complications (4\%): in one patient a transient wound healing disorder occurred which required no further surgical intervention. One patient showed postoperative aggravation of a preoperatively existing ataxia. There were no permanent complications. No patient showed haemorrhage on postoperative CT scan.

\section{Histopathological results}

Histological evaluation revealed pilocytic astrocytoma $(n=2)$, WHO grade II glioma $(n=14)$, malignant glioma $(n=12)$, metastasis $(n=7)$, lymphoma $(n=5)$ (eg, see fig 1$)$, cavernoma $(\mathrm{n}=1)$, inflammatory abscess $(\mathrm{n}=1)$ and an inflammatory demyelinising disease $(n=1)$. The diagnosis no tumour/gliosis was achieved in three patients (table 2). In this three cases, the stereotactic biopsy was repeated and showed an identical result.

\section{Correlation of histological and radiological findings}

Neuroradiological assessment as glioma differed from histopathological diagnosis in 14 of 46 patients. In the subgroup of patients with low grade gliomas (glioma grades I+II), the specificity of MRI was $46.6 \%$ and sensitivity was $62.5 \%$ (table 3 ). In the subgroup of patients harbouring glioma grade III or IV, the specificity of MRI was $61.7 \%$ and sensitivity was $58.3 \%$ (table 4 ).

For tumour grading, radiological assessments were correct in $7 / 20(35 \%)$ for low grade and in $7 / 26(27 \%)$ for high grade glioma.

Pathologies different from glioma showed much variety, ranging from other neoplastic diseases (lymphoma $(n=5)$, metastasis $(n=7))$ to benign lesions (cavernoma $(n=1)$, no tumour/gliosis $(n=3)$ ) to inflammatory disease (encephalitis disseminata, abscess $(\mathrm{n}=2)$ ) (table 2).

\section{Follow-up/length of survival}

All patients with no tumour/gliosis, inflammatory disease or pilocytic astrocytoma survived the entire period of observation or at least $\geqslant 1$ year. Patients with WHO grade II glioma showed a 1 year survival rate of $93 \%$ while this rate was $42 \%$ in patients with malignant glioma (fig 2). Patients with lymphoma or metastasis had a 1 year survival rate of $33 \%$.

Negative predictors for length of survival in patients with verified brainstem gliomas were higher tumour grade $(p=0.002)$ and Karnofsky performance score (KPS) ( $p=0.004)$. Age, duration of symptoms, cranial nerve impairment, level of the tumour,

Table 1 Baseline characteristics of the patients enrolled in the study

\begin{tabular}{ll}
\hline Characteristic & No \\
\hline Age (years) & $43(18-78)$ \\
Sex (M/F) & $25 / 21$ \\
Duration of symptoms (weeks) & $40(1-360)$ \\
Survival (months) & $28(1-91)$ \\
Follow-up (months) & $17(1-91)$ \\
\hline
\end{tabular}

Values are median (range) or number. 
Figure 1 A 66-year-old female. Symptoms developed within 3 weeks: hemiparesis, hemihypesthesia, facial palsy and vertigo. MRI showed contrast enhancing lesion on $T 1$ weighted $(A, B)$ and diffuse hyperintense lesion on T2 weighted (C) images. Histological workup revealed a high cellular density (haematoxylin-eosin (HE)) (D), a high proliferation index $(\mathrm{E})$ and strong immunoreactivity for CD79a (F). Histopathological diagnosis was B cell lymphoma.
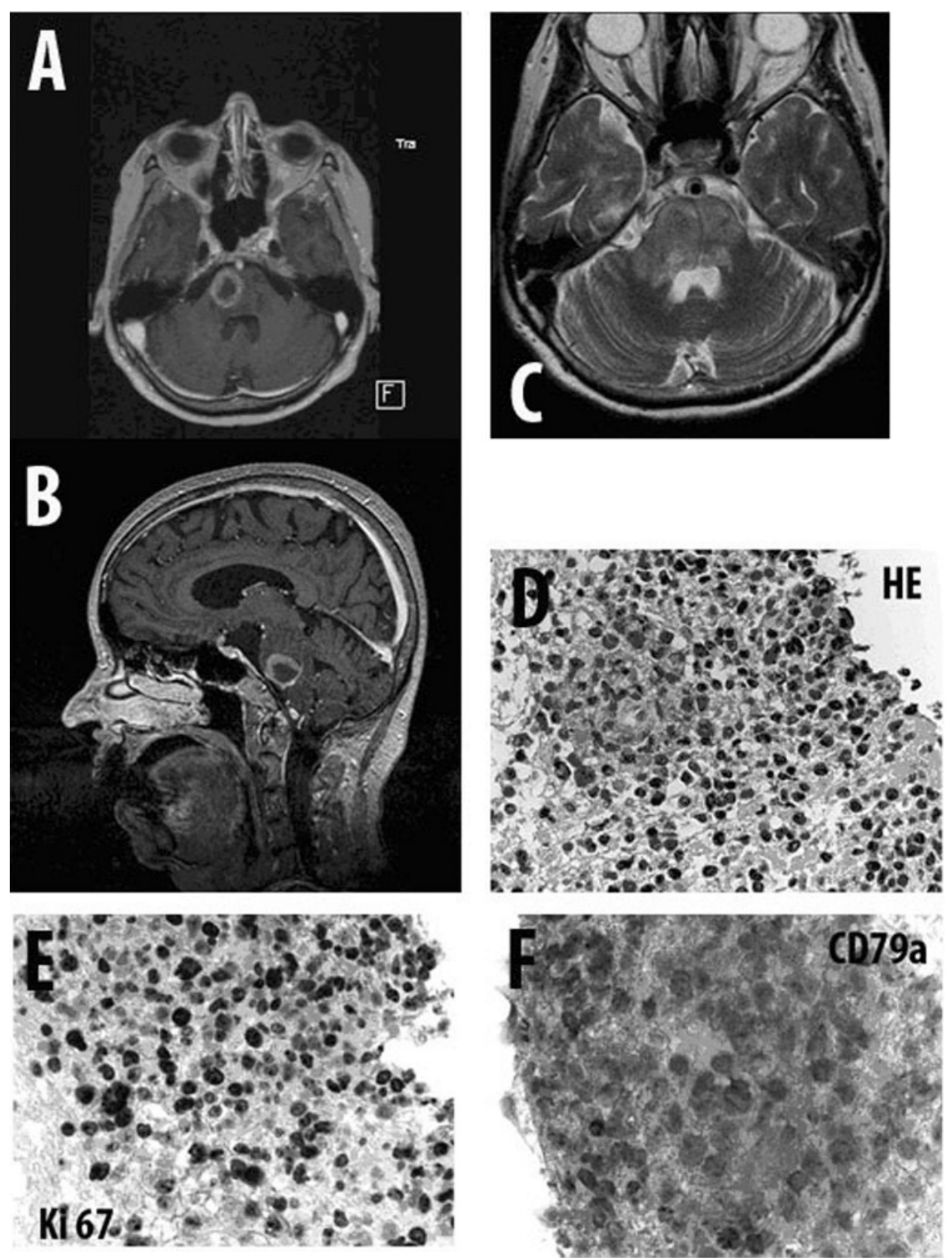

Table 2 Radiologically suspected brainstem glioma (WHO I ${ }^{\circ}-\mathrm{IV}^{\circ}$ )

\begin{tabular}{lc}
\hline Histology & No \\
\hline Pilocytic astrocytoma $\left(\mathrm{WHO} \mathrm{I}^{\circ}\right)$ & 2 \\
Low grade astrocytoma $\left(\mathrm{WHO} \mathrm{II}^{\circ}\right)$ & 14 \\
Anaplastic astrocytoma $\left(\mathrm{WHO} \mathrm{II}^{\circ}\right)$ & 8 \\
Glioblastoma multiforme $\left(\mathrm{WHO} \mathrm{IV}^{\circ}\right)$ & 4 \\
Metastasis & 7 \\
Lymphoma & 5 \\
Inflammation & 2 \\
Cavernoma & 1 \\
No tumour/gliosis & 3 \\
\hline
\end{tabular}

Histological results in 46 patients with radiologically suspected "glioma" in the brainstem. In 28/46 patients, radiological diagnosis was confirmed by histology.
Table 3 Radiologically suspected low grade gliomas $\left(\mathrm{WHO} \mathrm{I}^{\circ}\right.$ and $\left.\mathrm{II}^{\circ}\right)$

\begin{tabular}{ll}
\hline Histology & No \\
\hline Pilocytic astrocytoma & 1 \\
Low grade astrocytoma II & 6 \\
Anaplastic astrocytoma III & 4 \\
Glioblastoma multiforme & 3 \\
Lymphoma & 2 \\
Inflammation & 2 \\
No tumour/gliosis & 1 \\
Cavernoma & 1 \\
Metastasis & 6 \\
\hline Histological diagnosis in the subgroup of patients with suspected \\
low grade glioma. In seven of 26 patients, tissue diagnosis met \\
radiological assessment.
\end{tabular}


Table 4 Radiologically suspected high grade gliomas (WHO III $^{\circ}$ and IV ${ }^{\circ}$ )

\begin{tabular}{ll}
\hline Histology & No \\
\hline Pilocytic astrocytoma $\left(\mathrm{WHO} \mathrm{I}^{\circ}\right.$ ) & 1 \\
Low grade astrocytoma $\left(\mathrm{WHO} \mathrm{II}^{\circ}\right)$ & 6 \\
Anaplastic astrocytoma $\left(\mathrm{WHO} \mathrm{II}^{\circ}\right)$ & 5 \\
Glioblastoma multiforme $\left(\mathrm{WHO} \mathrm{IV}^{\circ}\right)$ & 2 \\
Lymphoma & 3 \\
No tumour/gliosis & 2 \\
Metastasis & 1 \\
\hline Histological diagnosis in the subgroup of patients with radiologically \\
suspected high grade glioma. Seven of 20 patients showed high \\
grade glioma in the histological workup.
\end{tabular}

multifocal extension, tumour delineation and contrast enhancement had no significant influence on length of survival (table 5). Multivariate analysis was not performed because of shortage of events.

\section{DISCUSSION}

In the present prospective study, we analysed the diagnostic value of conventional MRI based diagnosis for assessment of presumed intra-axial brainstem gliomas in an adult population by comparison with histopathological diagnosis. In this context, we further analysed the risk profile for stereotactic brainstem biopsies and identified prognostic factors for survival in these patients.

The diagnostic management of patients with brainstem tumours is controversial. Most authors rely on MR imaging for treatment decisions as radiological findings are considered to be sufficiently characteristic and specific. ${ }^{14}$ Thus histological diagnosis is not warranted in most studies as biopsy of brainstem lesions is considered to be a high risk procedure. ${ }^{11-13}$

In this study, patients were enrolled whose MRI images showed suspected brainstem glioma according to established features. ${ }^{14} 15$ Other brainstem lesions, which appeared radiologically untypical for gliomas, were not included in this prospective investigation.

\section{Diagnostic accuracy}

Biopsy of those lesions thought to be gliomas revealed a histopathological diagnosis different from the radiological assessment in 18 of 46 cases by means of differentiating gliomas from non-gliomas. This included two patients where the lesion was found to be of an inflammatory nature (with negative CSF parameters). In seven cases, metastasis of so far unknown origin was found instead of a glioma. In five patients, lymphoma was diagnosed as was a cavernoma in one patient. Three patients showed no tumour or inflammatory tissue but only reactive gliotic tissue, possibly linking to previous inflammatory of vascular incidents. Thus radiological assessment misleadingly classified some potential benign findings as gliomas. Considering the completely different treatment options for low grade glioma (brachytherapy, radiotherapy), high grade glioma (radiotherapy, chemotherapy), abscess (draining, intravenous antibiotics) and inflammatory lesion (immunosuppression, immunomodulation), the percentage of incorrect radiological diagnoses based on established features is extremely high. Thus incorrect treatment decisions in these patients may have fatal consequences or at least unnecessary strain.

In the group of radiological high grade gliomas, only seven of 20 patients had a glioma grade III or IV ${ }^{\circ}$ while 10 patients had low grade (grade I or II) glioma, lymphoma or metastasis. With regard to the therapeutic consequence of radiotherapy or radiochemotherapy, these patients might have gained some benefit from treatment but for two patients with no histological evidence of a tumour, this treatment based on the radiological diagnosis might have had severe consequences. A similar set was observed in the group of suspected low grade gliomas, where two of 26 patients showed inflammatory disease. Regarding this high number of incorrect classifications of brainstem lesions by radiological means and the necessity for highly specific therapies for the different pathological entities, MRI alone should not be used for treatment decisions, in our opinion.

In this study, all patients had a clear radiological diagnosis of a brainstem glioma. Even then, within this supposedly homogeneous group, this diagnosis was found to be incorrect in $60 \%$ of cases (eg, see fig 1). For suspected low grade gliomas, MRI specificity was as low as $46.6 \%$; for malignant gliomas it reached $61.7 \%$. However, we cannot exclude the fact that the explanatory power of these numbers might have been increased by the involvement of a second independent neuroradiologist.

Our findings may be interpreted as being in contrast with other studies published. Schumacher et al described a series of
Figure 2 Survival curve for study cohort. Kaplan-Meier was estimated for 46 patients within a $95 \%$ two sided confidence interval.

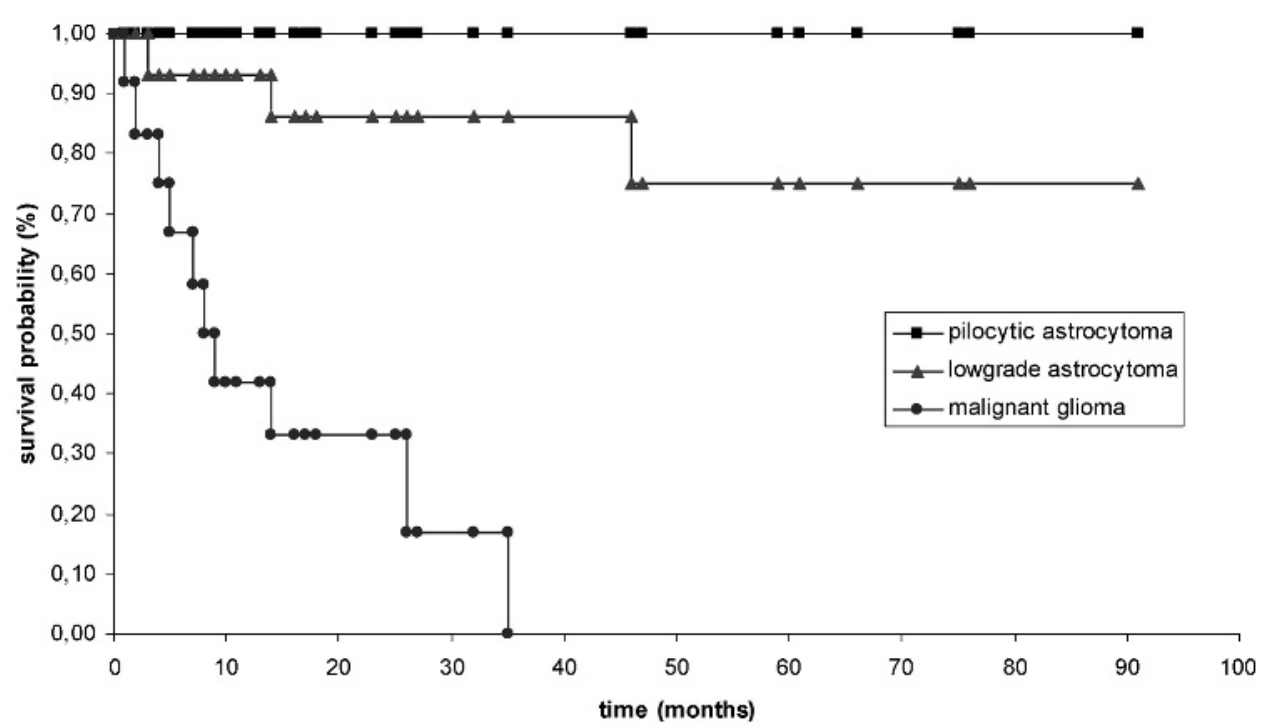


Table 5 Univariate analysis of survival

\begin{tabular}{ll}
\hline Candidate covariates & $\begin{array}{l}\text { Univariate } \\
\mathbf{p} \text { value }\end{array}$ \\
\hline Diffuse growth pattern & 0.96 \\
Extension $>1$ segment & 0.43 \\
Higher tumour grade & $0.002^{*}$ \\
Age & 0.15 \\
Duration of symptoms & 0.39 \\
Contrast enhancement & 0.21 \\
Karnofsky performance score & $0.004^{*}$ \\
Cranial nerve impairment & 0.85 \\
\hline
\end{tabular}

In univariate analysis, two prognostic factors $\left(^{*}\right)$ reached statistical significance for influence on survival.

142 paediatric cases with brainstem lesions, reporting a correct classification by the radiologist in up to $90 \% .{ }^{17}$ This may be due to the paediatric patient population in this study where a different frequency for various tumours is prevalent compared with adults. Furthermore, lesions such as metastasis, lymphoma or encephalitis disseminata are extremely rare in children and thus the histological variety of brainstem lesions is larger in adults, with important differences for therapeutic decisions.

For adult patient populations there are no recent studies comparing MRI findings with histological diagnosis in brainstem gliomas. The data of Kesari and colleagues ${ }^{10}$ include a high number of patients with brainstem tumours but histological data are provided for only $53 \%$. A recent study by Salmaggi and colleagues ${ }^{18}$ included 32 patients with histopathology data in 21 . Thus we have provided reliable data focusing on the accuracy of conventional MRI in the diagnosis of brainstem gliomas for the first time.

New imaging technologies such as MR spectroscopy and positron emission tomography have been proved to be useful tools for assessing supratentorial gliomas ${ }^{19-21}$ and may improve the non-invasive diagnosis of brainstem gliomas also, but prospective data regarding the accuracy of these methods in this subset of tumours are pending.

It should be noted that radiological assessment, even with inclusion of further imaging techniques, is subject to an individual range of interpretation, which is not only based on experience but also influenced by the amount of available additional information for the radiologist; a fact which also applies - to a lesser extent-to pathologists' assessment. Thus in the light of both the variety of pathologies frequently demanding specific treatment strategies and the justifiable procedure related risk, maximal yield of diagnostic information, including stereotactic biopsy, should be pursued.

Of further importance is the upcoming role of molecular markers in glioma therapy. ${ }^{22-26}$ As an example, in the treatment of malignant gliomas, MGMT promoter methylation has a growing impact on treatment decisions, as well as other molecules (eg, integrins). Although clinical data for brainstem gliomas and the impact of MGMT on treatment response are pending, the influence of molecular markers on treatment decisions might be significant and will require tissue diagnosis.

\section{Risk profile}

Although several authors showed a large variety of histological results in brainstem masses by brainstem biopsy, ${ }^{27-29}$ the indication for this procedure is still a matter of debate. Even in the most recent study by Kesari et al with a high number of patients, histological diagnosis was obtained in only $53 \%$ of cases. ${ }^{10}$ Many authors claim a very restrictive indication for radiologically unclear lesions because of the presumed high risk profile.
In some published studies, a high number of complications by stereotactic brainstem biopsies occurred, reaching up to $10 \% .{ }^{11-13}$ As a consequence, the authors of these studies propagated a non-invasive approach. ${ }^{12-14}$ Previous studies from our institution have proved that stereotactic biopsy is a safe and reliable method with a high diagnostic yield. ${ }^{30} 31$ In this present study, there were no major procedure related complications in spite of the highly eloquent target localisation. Apart from one wound healing disorder, only one patient showed a slight deterioration of a preoperatively existing ataxia, which recovered slowly during the following months. There were no procedure related bleedings. The extremely low incidence of complications in the present study may be attributed to a very high number of stereotactic biopsies per year at the authors' institution and a highly standardised workflow. Thus our numbers may not reflect the actual risk profile at centres with less experience of stereotactic procedures.

The diagnostic results obtained by biopsy were conclusive with follow-up data. Three patients with no pathological findings in the first biopsy underwent re-biopsy with an identical histological pattern. The natural course of these patients underlines the absence of a tumour or inflammatory disease and thus confirmed the histological diagnosis.

Comparing the reported procedure related risk of a stereotactic biopsy in suspected brainstem glioma with the probability of an incorrect radiological diagnosis and its therapeutic consequences, biopsy not only is justifiable but should be pursued in these patients, in our opinion.

Nevertheless, brainstem lesions are comparatively rare findings. Thus a successful and safe biopsy in brainstem regions requires a high technical infrastructure, an experienced stereotactic neurosurgeon and a highly standardised workflow for these procedures. Thus biopsies in these eloquent areas should be performed in specialised centres.

\section{Prognostic factors for survival}

There are few current data dealing with the prognosis and prognostic factors in brainstem gliomas in adults. ${ }^{10} 32$ This may be because of the low incidence of these tumours and rarely confirmed histopathological diagnosis. In our series, only tumour grade $(p=0.002)$ and KPS $(p=0.004)$ reached statistical significance. In the most recent study by Kesari and colleagues, ${ }^{10}$ a large series of brainstem tumours in adults was evaluated retrospectively with regard to prognostic factors. In Kesari's series several other parameters (eg, age and duration of symptoms) also reached statistical significance. Furthermore, survival rate for low grade gliomas in his study was significantly larger than in our series. However, in Kesari's study, histological diagnosis was obtained in only 54 of 101 patients. Considering the limited diagnostic accuracy of MRI for glioma diagnosis, these data may be influenced by a certain number of non-glioma lesions which we have shown to have a completely different natural course.

\section{CONCLUSION}

The diagnostic value of conventional MRI imaging in treatment planning of brainstem gliomas in adults is insufficient. Tissue diagnosis is mandatory for adequate treatment decision. Multimodally guided stereotactic biopsy of brainstem gliomas is a low risk procedure with a high diagnostic value in experienced hands and thus should be regarded as standard in adult patients with brainstem lesions.

Competing interests: None.

Provenance and peer review: Not commissioned; externally peer reviewed. 


\section{REFERENCES}

1. Laigle-Donadey F, Doz F, Delattre JY. Brainstem gliomas in children and adults. Curr Opin Oncol 2008;20:662-7.

2. Chang A, Merchant T. Patterns of failure for diffuse infiltrating brainstem glioma: New guidelines for radiotherapy planning. Neuro-oncol 2008;10:392.

3. Piette C, Deprez M, Born J, et al. Management of diffuse glioma in children: a retrospective study of 27 cases and review of literature. Acta Neurol Belg 2008; 108:35-43.

4. Frappaz D, Schell M, Thiesse $P$, et al. Preradiation chemotherapy may improve survival in pediatric diffuse intrinsic brainstem gliomas: Final results of BSG 98 prospective trial. Neuro-oncol 2008;10:599-607.

5. Haas-Kogan DA, Banerjee A, Kocak M, et al. Phase I trial of tipifarnib in children with newly diagnosed intrinsic diffuse brainstem glioma. Neuro-oncol 2008;10:341-7.

6. Goldman S, Tomita T, Marymont M, et al. Phase II trial thalidomide and carboplatin for the treatment of brainstem glioma (CNS1099). Neuro-oncol 2008;10:392-3.

7. Shah NC, Ray A, Bartels U, et al. Diffuse intrinsic brainstem tumors in neonates J Neurosurg Pediatr 2008;1:382-5.

8. Pavelka Z, Zitterbart K, Pavlik T, et al. Diffuse brainstem gliomas in children. A nightmare for a paediatric oncologist. Ceska A Slovenska Neurol Neurochirurg 2008;71:41-6.

9. Fouladi M, Nicholson HS, Zhou T, et al. A phase II study of the farnesyl transferase inhibitor, tipifarnib, in children with recurrent or progressive high-grade glioma, medulloblastoma/primitive neuroectodermal tumor, or brainstem glioma- $\mathrm{A}$ children's oncology group study. Cancer 2007;110:2535-41.

10. Kesari S, Kim RS, Markos V, et al. Prognostic factors in adult brainstem gliomas: a multicenter, retrospective analysis of 101 cases. J Neuro-oncol 2008;88:175-83.

11. Guillamo JS, Doz F, Delattre JY. Brain stem gliomas. Curr Opin Neurol 2001;14:711-15.

12. Albright AL, Packer RJ, Zimmerman R, et al. Magnetic-resonance scans should replace biopsies for the diagnosis of diffuse brain-stem gliomas - a report from the Childrens Cancer Group. Neurosurgery 1993;33:1026-30.

13. Albright AL. Diffuse brainstem tumors: When is a biopsy necessary? Pediatr Neurosurg 1996;24:252-5.

14. Donaldson SS, Laningham F, Fisher PG. Advances toward an understanding of brainstem gliomas. J Clin Oncol 2006;24:1266-72.

15. Fischbein NJ, Prados MD, Wara W, et al. Radiologic classification of brain stem tumors: Correlation of magnetic resonance imaging appearance with clinical outcome. Pediatr Neurosurg 1996;24:9-23.

16. Kreth FW, Faist M, Warnke PC, et al. Stereotaxic biopsy for nonpilocytic astrocytomas. J Neurosurg 1995;83:759-60.
17. Schumacher $\mathbf{M}$, Schulte-Monting J, Stoeter $\mathbf{P}$, et al. Magnetic resonance imaging compared with biopsy in the diagnosis of brainstem diseases of childhood: a multicenter review. J Neurosurg 2007;106:111-19.

18. Salmaggi A, Fariselli L, Milanesi I, et al. Natural history and management of brainstem gliomas in adults-A retrospective Italian study. J Neurol 2008:255:171-7.

19. Langen KJ, Floeth FW, Stoffels G, et al. Improved diagnostics of cerebral gliomas using FET PET. Z Med Phys 2007;17:237-41.

20. Mehrkens JH, Popperl G, Rachinger W, et al. The positive predictive value of 0-(2 [F-18]fluoroethyl)-L-tyrosine (FET) PET in the diagnosis of a glioma recurrence after multimodal treatment. J Neuro-oncol 2008;88:27-35.

21. Rachinger W, Goetz C, Popperl G, et al. Positron emission tomography with 0-(2-[F18]fluoroethyl)-L-tyrosine versus magnetic resonance imaging in the diagnosis of recurrent gliomas. Neurosurgery 2005;57:505-11.

22. Hegi ME, Liu LL, Herman JG, et al. Correlation of 0-6-methylguanine methyltransferase (MGMT) promoter methylation with clinical outcomes in glioblastoma and clinical strategies to modulate MGMT activity. J Clin Oncol 2008;26:4189-99.

23. Paus C, Murat A, Stupp R, et al. Role of MGMT and clinical applications in brain tumours. Bull Cancer 2007;94:769-73.

24. van den Bent MJ, Hegi ME, Stupp R. Recent developments in the use of chemotherapy in brain tumours. Eur J Cancer 2006;42:582-8.

25. Norden AD, Drappatz J, Wen PY. Antiangiogenic therapy in malignant gliomas. Curr Opin Oncol 2008;20:652-61.

26. Reardon DA, Nabors LB, Stupp R, et al. Cilengitide: an integrin-targeting arginineglycine-aspartic acid peptide with promising activity for glioblastoma multiforme. Expert Opin Investig Drugs 2008;17:1225-35.

27. de Leon FCP, Perezpena-Diazconti M, Castro-Sierra E, et al. Stereotactically-guided biopsies of brainstem tumors. Childs Nervous System 2003;19:305-10.

28. Goncalves-Ferreira AJ, Herculano-Carvalho M, Pimentel J, et al. Stereotactic biopsies of focal brainstem lesions. Surg Neurol 2003;60:311-20.

29. Pincus DW, Richter E0, Yachnis AT, et al. Brainstem stereotactic biopsy sampling in children. J Neurosurg 2006;104:108-14.

30. Kreth FW, Muacevic A. Stereotactic biopsy and hemorrhage. J Neurosurg 1999;90:181-2.

31. Kreth FW, Muacevic A, Medele R, et al. The risk of haemorrhage after image guided stereotactic biopsy of intra-axial brain tumours-A prospective study. Acta Neurochirurg 2001;143:539-45.

32. Sousa P, Hinojosa J, Munoz M, et al. Brainstem gliomas. Neurocirugia 2004;15:56-66 


\section{JNNP}

\section{Serial stereotactic biopsy of brainstem lesions in adults improves diagnostic accuracy compared with MRI only}

W Rachinger, S Grau, M Holtmannspötter, et al.

J Neurol Neurosurg Psychiatry 2009 80: 1134-1139 originally published online June 10, 2009

doi: 10.1136/jnnp.2009.174250

Updated information and services can be found at:

http://jnnp.bmj.com/content/80/10/1134.full.html

\begin{tabular}{|c|c|}
\hline & These include: \\
\hline \multirow[t]{2}{*}{ References } & $\begin{array}{l}\text { This article cites } 32 \text { articles, } 4 \text { of which can be accessed free at: } \\
\text { http://jnnp.bmj.com/content/80/10/1134.full.html\#ref-list-1 }\end{array}$ \\
\hline & $\begin{array}{l}\text { Article cited in: } \\
\text { http://jnnp.bmj.com/content/80/10/1134.full.html\#related-urls }\end{array}$ \\
\hline $\begin{array}{l}\text { Email alerting } \\
\text { service }\end{array}$ & $\begin{array}{l}\text { Receive free email alerts when new articles cite this article. Sign up in } \\
\text { the box at the top right corner of the online article. }\end{array}$ \\
\hline \multirow{2}{*}{$\begin{array}{r}\text { Topic } \\
\text { Collections }\end{array}$} & Articles on similar topics can be found in the following collections \\
\hline & $\begin{array}{l}\text { CNS cancer (147 articles) } \\
\text { Neurooncology (199 articles) } \\
\text { Radiology (1436 articles) } \\
\text { Surgical diagnostic tests (320 articles) } \\
\text { Immunology (including allergy) (1453 articles) }\end{array}$ \\
\hline
\end{tabular}

Notes

To request permissions go to:

http://group.bmj.com/group/rights-licensing/permissions

To order reprints go to:

http://journals.bmj.com/cgi/reprintform

To subscribe to BMJ go to:

http://group.bmj.com/subscribe/ 